\title{
Preface: Hydrology of Large River Basins in Africa
}

\author{
Ernest Amoussou $^{1,2,3,4}$, Gil Mahe ${ }^{3,4,5}$, Oula Amrouni ${ }^{3,6}$, Ansoumana Bodian ${ }^{7}$, Christophe Cudennec ${ }^{4,8}$, \\ Stephan Dietrich $^{3,9}$, Domiho Japhet Kodja ${ }^{2}$, and Expédit Wilfrid Vissin ${ }^{2}$ \\ ${ }^{1}$ Université de Parakou, Parakou, Bénin \\ ${ }^{2}$ Laboratoire Pierre Pagney: Climate, Water, Ecosystems and Development (LACEEDE), \\ Université d'Abomey-Calavi, Cotonou, Bénin \\ ${ }^{3}$ FRIEND-Water program, Intergovernmental Hydrological Program, \\ UNESCO/Flow Regimes from International, Experimental and Network Data, Paris, France \\ ${ }^{4}$ International Association of Hydrological Sciences, Wallingford, UK \\ ${ }^{5}$ HSM, Université Montpellier, IRD, CNRS, IMT, Case MSE, \\ 163 rue Auguste Broussonnet, 34090, Montpellier, France \\ ${ }^{6}$ Institut National des Sciences et Technologies de la Mer, Salammbô, Université de Carthage, Tunis, Tunisie \\ ${ }^{7}$ Laboratoire Leïdi "Dynamique des territoires et développement”, Université Gaston Berger (UGB), \\ BP 234-Saint-Louis, Sénégal \\ ${ }^{8}$ UMR SAS, Institut Agro, INRAE, Rennes, France \\ ${ }^{9}$ International Centre for Water Resources and Global Change, \\ Federal Institute of Hydrology, Koblenz, Germany \\ Correspondence: Ernest Amoussou (ernestamoussou@gmail.com) \\ and Gil Mahe (gil.mahe@ird.fr) \\ Published: 16 November 2021
}

FRIEND-Water is a flagship program of the UNESCO Intergovernmental Hydrological Program. Since it started in 1985 the International Association of Hydrological Sciences (IAHS) gave its scientific support to the every 4 years global FRIEND conferences and some other occasional issues, which resulted in the publication of previously called "redbooks" (available online at https://iahs.info, last access: 18 October 2021), and now Proceedings of IAHS (PIAHS). This collaboration between FRIEND-Water and IAHS is very important to keep developing the visibility and attractiveness of hydrological and related sciences in all countries of the World, particularly in developing countries. This PI$A H S$ issue publishes articles presented at the 4th International Conference on the Hydrology of Large Rivers Basins of Africa, organized by hydrologists of Benin. The co-editors of this issue come from Benin, Senegal, Tunisia, Germany and France, which shows the strong South-North relationships between institutions and colleagues. From more than 230 abstracts, 132 papers were submitted to the PIAHS issue, and 56 were eventually accepted after a double peerreviewing process involving 122 reviewers from 21 countries, listed below.
This conference is the fourth of a series started in 2015 in Tunisia, followed by a second edition in Senegal in 2016, and a third one in Algeria in 2018. Scheduled in 2020 according to the 2-years pace, it has been delayed due to the covid crisis, but is organized mainly with physical presence in November 2021 in Cotonou, Benin. As usual this conference is the occasion to organize training courses, side-event workshops, and for the first time a PIAHS volume. This cycle of conferences also refers to the World Large Rivers Initiative launched by UNESCO in 2014 to which it contributes for the continent. The scientific coverage is comprehensive across the global theme of large rivers; ranging from observation, characterisation and modelling of various processes and settings, to the analysis of regimes, changes and security issues. Articles published in that issue, are hence much related to most of the 10 International Commissions of IAHS, and to cross-cutting and agenda-setting initiatives: innovation and assimilation in hydrometry and the data-service value chain (Tauro et al., 2018; Cudennec et al., 2020; in consistence with Pecora and Lins, 2020 and Dixon et al., 2021), Prediction in Ungauged Basins - PUB (Hrachowitz et al., 2013), Hydrological change - Panta Rhei (Montanari et al., 2013; 
McMillan et al., 2016) and related Water security (Young et al., 2015; Ceola et al., 2016) and nexus approach (Liu et al., 2017; Connor et al., 2020; Heal et al., 2021), and socio-hydrology and citizen engagement (Di Baldassarre et al., 2019; Nardi et al., 2021). The clustering of the articles in three sections captures that alignment through a progression from hydrological dynamics, to regimes and changes, and to security. The range of African Large Rivers addressed across these articles is a richness as such, with an ultimate value for comparative hydrology; whereas some local studies are also included, which are valuable for documenting knowledge and models.

This open access (as all PIAHS volumes) bilingual issue is of particular interest and value for a large community of authors and readers in Africa, in terms of knowledge capitalization and sharing, both at the regional and international levels, within academia and in the science-policy bridging as advocated by learned societies and UNESCO in terms of Open Science and public good (Cudennec and Hubert, 2008; Hughes et al., 2014; Quinn et al., 2018; Boulton et al., 2021; UNESCO, 2021). Leading authors come from 15 African countries, Canada, France and UK. Beyond these figures, this issue shows the great expectation of African researchers to integrate international institutional activities and share common scientific reflections. This PIAHS volume, as a contribution to the FRIEND-Water program, shortly follows the one published in 2020 out of the Beijing Global FRIENDWater conference in 2018 (Yu et al., 2020). The next Global FRIEND-Water conference is scheduled to be held in Dakar, Senegal in March 2023. The contribution of the African scientific community to IAHS activities is strongly increasing (Hughes et al., 2015; Croke and Jewitt, 2018; Makurira et al., 2018), which is also visible by the very large registration of African colleagues to the ongoing 23 UPHs in Africa initiative (Blöschl et al., 2019), whose results should be integrated to the global 23 UPHs discussions scheduled in Montpellier IAHS XIth scientific assembly in May-June 2022.

Acknowledgements. Many contributors helped funding this issue and the conference, Institutions: UNESCO Intergovernmental Hydrological Program, the International Centre for Water Resources and Global Change of Koblenz, Germany, IAHS including its SYSTA granting mechanism, the Research Institute for Development - IRD France, the World Meteorological Organization, l'Agence Universitaire de la Francophonie, le CRASTE-LF Centre de Recherche Africain des Sciences et Technologies de l'Espace en Langue Française Maroc, Ministère de l'Enseignement Supérieur et de la Recherche Scientifique du Bénin, Ministère de l'Eau et des Mines du Bénin, Ministère du Cadre de Vie et du Développement Durable du Bénin, Ministère de l'Agriculture et de l'Alimentation du Bénin, Ambassade de France au Bénin, Ambassade de France au Congo, Agence Française de Développement, Académie Nationale des Sciences Arts et Lettres du Bénin, AHR Foundation Bénin; and Universities and Laboratories: University of Parakou Bénin, University of Abomey-Calavi Bénin, Institut de Géogra- phie de l'Aménagement du Territoire et de l'Environnement, Institut National de l'Eau, Laboratoire Pierre Pagney Climat, Ecosystème, Eau et Développement, Laboratoire GéoSciences Environnement Toulouse France, Laboratoire HydroSciences Montpellier France. The following hydrologists have contributed as reviewers: Abderrahmane Khedimallah, Abderrazak Bouanani, Larbi Djabri, Ahmed Kettab, Amin Zettam, Abdelhadi Ammari, Ayoub Zeroual, Belkacem Bekkoussa, Faiza Hallouz, Hind Meddi, Mohamed Meddi, Ayoub Zeroual, Sabrina Taibi, Samir Toumi, SidAhmed Bouguerra, from Algeria; Dawit Berhane from Australia; Firmin Adandedji, Constant Houndenou, K. Olive Adissoda, Fidèle Ahehehinnou Yedo, Gervais Atchade, Djigbo Félicien Badou, Eliezer Biao, Michel Boko, Aymar Bossa, Cyr Gervais Etene, Eric A. Alamou, Moussa Gibigaye, Jean Hounkpe, Domiho Japhet Kodja, Olivier Koudamiloro, Hervé Koumassi, Maurice Ahouansou, Brice Mongbo, Pierre I. Akponikpe, Maïeul G. Quenum, S. Henri Totin Vodounon, Expédit Wilfrid Vissin, Ibouraïma Yabi, Josué Zandagba, Salou Zoulkif, Wilfrid Expédit Vissin, Ernest Amoussou, Rafiath Bamisso, N. P. Maximilien Boko, from Benin; Yetchékpo Patrick Gbohoui, Issoufou Ouedraogo, Yacouba Yira, Augustin Yameogo from Burkina-Faso; Wilfried Pokam, Jules Rémy Ndam, Rodric Merime Nonki, Elisabeth Fita Dassou from Cameroon; Yangbo Chen from China; Martin Kabantu from Congo; François Assemian, Modeste Kacou, Kouakou Lazare Kouassi, Auguste Kouassi Kouame, Amani Michel Kouassi, Djibril Dabissi Noufe, Salomon Obahoundje, Zamblé Armand Tra Bi, Vami Nguessan, Mouamé Y. Morton, Eric Pascal Zahiri from Cöte D’Ivoire; Negash Tessema from Ethiopia; Alain Dezetter, Andrew Ogilvie, Benjamin Polh, Pascal Breil, Charles Perrin, Christophe Bouvier, Christophe Brachet, Christophe Cudennec, Daniel Loudiere, Olivier Delaigue, Didier Orange, Arona Diedhiou, Emmanuelle Cadot, Gil Mahe, Jean-Louis Perrin, Jean Emmanuel Paturel, Sandra Rome, Alban Delavenne, Sylvain Bigot, Manuella Grippa, Yves Tramblay, Valentin Wendling, Théo Vischel, Telesphore Brou, Etienne Guilpart, Vazken Andreassian from France; Felix Opdehipt, Jörg Dietrich, Stephan Dietrich from Germany; Lhoussaine Bouchaou from Morocco; Nicolas Vigaud from USA; Olusegun Adeaga, Isaiah Akoteyon from Nigeria; Cyriaque Rufin Nguimalet from Central African Republic; Mamadou Lamine Mbaye, Soussou Sambou, Raymond Malou, Amadou Abou Sy, Abdoulaye Faty, Ibrahima Mall, Lamine Diop, Gayane Faye, Assoumana Bodian from Senegal; Lamboni Batablinle, Benoît Koubodana from Togo; Sihem Benabdallah, Noureddine Gaaloul, Majid Mathlouthi, Oula Amrouni from Tunisia; Nilay Dogulu from Turkey; Declan Conway, Bastien Dieppois, Maria Uberia, Abdou Khouakhi from United Kingdom.

\section{References}

Blöschl, G., Bierkens, M. F. P., Chambel, A., Cudennec, C., Destouni, G., Fiori, A., Kirchner, J. W., McDonnell, J. J., Savenije, H. H. G., Sivapalan, M., Stump, C., Toth, E., Volpi, E., Carr, G., Lupton, C., Salinas, J., Széles, B., Viglione, A., Aksoy, H., Allen, S. T., Amin, A., Andréassian, V., Arheimer, B., Aryal, S. K., Baker, V., Bardsley, E., Barendrecht, M. H., Bartosova, A., Batelaan, O., Berghuijs, W. R., Beven, K., Blume, T., Bogaard, T., Borges de Amorim, P., Böttcher, M. E., Boulet, G., Breinl, K., Brilly, M., Brocca, L., Buytaert, W., Castellarin, 
A., Castelletti, A., Chen, X., Chen, Y., Chen, Y., Chifflard, P., Claps, P., Clark, M. P., Collins, A. L., Croke, B., Dathe, A., David, P. C., de Barros, F. P. J., de Rooij, G., Di Baldassarre, G., Driscoll, J. M., Duethmann, D., Dwivedi, R., Eris, E., Farmer, W. H., Feiccabrino, J., Ferguson, G., Ferrari, E., Ferraris, S., Fersch, B., Finger, D., Foglia, L., Fowler, K., Gartsman, B., Gascoin, S., Gaume, E., Gelfan, A., Geris, J., Gharari, S., Gleeson, T., Glendell, M., Gonzalez Bevacqua, A., González-Dugo, M. P., Grimaldi, S., Gupta, A. B., Guse, B., Han, D., Hannah, D., Harpold, A., Haun, S., Heal, K., Helfricht, K., Herrnegger, M., Hipsey, M., Hlaváciková, H., Hohmann, C., Holko, L., Hopkinson, C., Hrachowitz, M., Illangasekare, T. H., Inam, A., Innocente, C., Istanbulluoglu, E., Jarihani, B., Kalantari, Z., Kalvans, A., Khanal, S., Khatami, S., Kiesel, J., Kirkby, M., Knoben, W., Kochanek, K., Kohnová, S., Kolechkina, A., Krause, S., Kreamer, D., Kreibich, H., Kunstmann, H., Lange, H., Liberato, M. L. R., Lindquist, E., Link, T., Liu, J., Loucks, D.P., Luce, C., Mahé, G., Makarieva, O., Malard, J., Mashtayeva, S., Maskey, S., Mas-Pla, J., Mavrova-Guirguinova, M., Mazzoleni, M., Mernild, S., Misstear, B. D., Montanari, A., Müller-Thomy, H., Nabizadeh, A., Nardi, F., Neale, C., Nesterova, N., Nurtaev, B., Odongo, V. O., Panda, S., Pande, S., Pang, Z., Papacharalampous, G., Perrin, C., Pfister, L., Pimentel, R., Polo, M. J., Post, D., Prieto Sierra, C., Ramos, M.-H., Renner, M., Reynolds, J. E., Ridolfi E., Rigon, R., Riva, M., Robertson, D.E., Rosso, R., Roy, T., Sá, J. H. M., Salvadori, G., Sandells, M., Schaefli, B., Schumann, A., Scolobig, A., Seibert, J., Servat, E., Shafiei, M., Sharma, A., Sidibe, M., Sidle, R. C., Skaugen, T., Smith, H., Spiessl, S. M., Stein, L., Steinsland, I., Strasser, U., Su, B., Szolgay, J., Tarboton, D., Tauro, F., Thirel, G., Tian, F., Tong, R., Tussupova, K., Tyralis, H., Uijlenhoet, R., van Beek, R., van der Ent, R.J., van der Ploeg, M., Van Loon, A.F., van Meerveld, I., van Nooijen, R., van Oel, P. R., Vidal, J.-P., von Freyberg, J., Vorogushyn, S., Wachniew, P., Wade, A. J., Ward, P., Westerberg, I. K., White, C., Wood, E. F., Woods, R., Xu, Z., Yilmaz, K. K., and Zhang, Y.: Twenty-three unsolved problems in hydrology (UPH) - a community perspective, Hydrolog. Sci. J., 64, 11411158, https://doi.org/10.1080/02626667.2019.1620507, 2019.

Boulton, G., Cudennec, C, Castle, D., Dogulu, N., Haliwell, J., Hélein, F., Maras, P., Rossini, M., Sanchez, J. A., Sud, M., and Vigen J.: ISC Statement on Open Science and the UNESCO initiative, available at: https://council.science/current/ news/open-science-and-the-unesco-initiative/, last access: 5 October 2021.

Ceola, S., Montanari, A., Krueger, T., Dyer, F., Kreibich, H. ,Westerberg, I., Carr, G., Cudennec, C., Elshorbagy, A., Savenije, H., Van Der Zaag, P., Rosbjerg, D., Aksoy, H., Viola, F., Petrucci, G., MacLeod, K., Croke, B., Ganora, D., Hermans, L., Polo, M.J., Xu, Z., Borga, M., Helmschrot, J., Toth, E., Ranzi, R., Castellarin, A., Hurford, A., Brilly, M., Viglione, A., Blöschl, G., Sivapalan, M., Domeneghetti, A., Marinelli, A., and Di Baldassarre, G.: Adaptation of water resources systems to changing society and environment - A statement by the International Association of Hydrological Sciences, Hydrolog. Sci. J., 61, 2803-2817, https://doi.org/10.1080/02626667.2016.1230674, 2016.

Connor, R., Timmerman, J., Uhlenbrook, S., and Koncagül, E.: Chapter 9 - Water-Climate-Energy-Food-Environment Nexus, The United Nations World Water Development Report 2020 "Water and Climate Change", 118-125, ISBN
978-92-3-100371-4, available at: https://en.unesco.org/themes/ water-security/wwap/wwdr/2020 (last access: 5 October 2021), 2020.

Croke, B. and Jewitt, G.: Editorial: Special Issue on Water security and the food-water-energy nexus: drivers, responses and feedbacks at local to global scales, Proc. IAHS, 376, 1-1, https://doi.org/10.5194/piahs-376-1-2018, 2018.

Cudennec, C. and Hubert, P.: Multi-objective role of HSJ in processing and disseminating hydrological knowledge, Hydrolog. Sci. J., 53, 485-487, https://doi.org/10.1623/hysj.53.2.485, 2008.

Cudennec, C., Lins, H., Uhlenbrook, S., and Arheimer, B.: Towards FAIR and SQUARE hydrological data, Hydrolog. Sci. J., 65, 681-682, https://doi.org/10.1080/02626667.2020.1739397, 2020.

Di Baldassarre, G., Sivapalan, M., Rusca, M., Cudennec, C., Garcia, M., Kreibich, H., Konar, M., Mondino, E., Mård, J., Pande, S., Sanderson, M. R., Tian, F., Viglione, A., Wei, J., Wei, Y., Yu, D. J., Srinivasan, V., and Blöschl, G.: Sociohydrology: Scientific challenges in addressing the sustainable development goals, Water Resour. Res., 55, 6327-6355, https://doi.org/10.1029/2018WR023901, 2019.

Dixon, H., Sandström, S., Cudennec, C., Lins, H.F., Abrate, T., Bérod, D., Chernov, I., Ravalitera, N., Sighomnou, D., and Teichert, F.: Intergovernmental cooperation for hydrometry - what, why, how?, Hydrolog. Sci. J., 1-15, https://doi.org/10.1080/02626667.2020.1764569, 2021.

Heal, K. V., Bartosova, A., Hipsey, M. R., Chen, X., Buytaert, W., Li, H.-Y., McGrane, S. J., Gupta, A. B., and Cudennec C.: Water quality: the missing dimension of water in the water-energy-food nexus, Hydrolog. Sci. J., 66, 745-758, https://doi.org/10.1080/02626667.2020.1859114, 2021.

Hrachowitz, M., Savenije, H. H. G., Blöschl, G., McDonnell, J. J., Sivapalan, M., Pomeroy, J. W., Arheimer, B., Blume, T., Clark, M. P., Ehret, U., Fenicia, F., Freer, J. E., Gelfan, A., Gupta, H. V., Hughes, D. A., Hut, R. W., Montanari, A., Pande, S., Tetzlaff, D., Troch, P. A., Uhlenbrook, S., Wagener, T., Winsemius, H.C., Woods, R.A., Zehe, E., and Cudennec, C.: A decade of Predictions in Ungauged Basins (PUB) - a review, Hydrolog. Sci. J., 58, 1198-1255, https://doi.org/10.1080/02626667.2013.803183, 2013.

Hughes, D. A., Heal, K. V., and Leduc, C.: Improving the visibility of hydrological sciences from developing countries, Hydrolog. Sci. J., 59, 1627-1635, https://doi.org/10.1080/02626667.2014.938653, 2014.

Hughes, D. A., Jewitt, G., Mahe, G., Mazvimavi, D., and Stisen, S.: A review of hydrological sciences research in Africa over the last decade, Hydrolog. Sci. J., 60, 1865-1879, https://doi.org/10.1080/02626667.2015.1072276, 2015.

Liu, J., Yang, H., Cudennec, C., Gain, A. K., Hoff, H., Lawford, R., Qi, J., de Strasser, L., Yillia, P. T., and Zheng, C.: Challenges in operationalizing the waterenergy-food nexus, Hydrolog. Sci. J., 62, 1714-1720, https://doi.org/10.1080/02626667.2017.1353695, 2017.

Makurira, H., Mazvimavi, D., Kapangaziwiri, E., Kileshye Onema, J.-M., and Gumindoga, W.: Preface: Understanding spatiotemporal variability of water resources and the implications for IWRM in semi-arid eastern and southern Africa, Proc. IAHS, 378, 1-1, https://doi.org/10.5194/piahs-378-1-2018, 2018. 
McMillan, H., Montanari, A., Cudennec, C., Savenije, H., Kreibich, H., Krueger, T., Liu, J., Mejia, A., Van Loon, A., Aksoy, H., D, Baldassarre, G., Huang, Y., Mazvimavi, D., Rogger, M., Sivakumar, B., Bibikova, T., Castellarin, A., Chen, Y., Finger, D., Gelfan, A., Hannah, D. M., Hoekstra, A. Y., Li, H., Maskey, S., Mathevet, T., Mijic, A., Acuña, A. P., Polo, M. J., Rosales, V., Smith, P., Viglione, A., Srinivasan, V., Toth, E., van Nooyen, R., and Xia, J.: Panta Rhei 2013-2015: Global perspectives on hydrology, society and change, Hydrolog. Sci. J., 61, 1174-1191, https://doi.org/10.1080/02626667.2016.1159308, 2016.

Montanari, A., Young, G., Savenije, H. H. G., Hughes, D., Wagener, T., Ren, L.L., Koutsoyiannis, D., Cudennec, C., Toth, E., Grimaldi, S., Blöschl, G., Sivapalan, M., Beven, K., Gupta, H., Hipsey, M., Schaefli, B., Arheimer, B., Boegh, E., Schymanski, S. J., Di, Baldassarre, G., Yu, B., Hubert, P., Huang, Y., Schumann, A., Post, D. A., Srinivasan, V., Harman, C., Thompson, S., Rogger, M., Viglione, A., McMillan, H., Characklis, G., Pang, Z., and Belyaev, V.: "Panta Rhei - Everything Flows": Change in hydrology and society - The IAHS Scientific Decade 2013-2022, Hydrolog. Sci. J., 58, 1256-1275, https://doi.org/10.1080/02626667.2013.809088, 2013.

Nardi, F., Cudennec, C., Abrate, T., Allouch, C., Annis, A., Assumpção, T., Aubert, A. H., Bérod, D., Braccini, A. M., Buytaert, W., Dasgupta, A., Hannah, D.M., Mazzoleni, M., Polo, M. J., Sæbø, Ø., Seibert, J., Tauro, F., Teichert, F., Teutonico, R., Uhlenbrook, S., Wahrmann Vargas, C. and Grimaldi, S.: Citizens AND HYdrology (CANDHY): conceptualizing a transdisciplinary framework for citizen science addressing hydrological challenges, Hydrolog. Sci. J., https://doi.org/10.1080/02626667.2020.1849707, in press, 2021.

Pecora, S. and Lins, H. F.: E-monitoring the nature of water, Hydrolog. Sci. J., 65, 683-698, https://doi.org/10.1080/02626667.2020.1724296, 2020.
Quinn, N., Blöschl, G., Bárdossy, A., Castellarin, A., Clark, M., Cudennec, C., Koutsoyiannis, D., Lall, U., Lichner, L., Parajka, J., Peters-Lidard, C. D., Sander, G., Savenije, H., Smettem, K., Vereecken, H., Viglione, A., Willems, P., Wood, A., Woods, R., $\mathrm{Xu}, \mathrm{C} .-$ Y., and Zehe, E.: Joint editorial: Invigorating hydrological research through journal publications, Hydrol. Earth Syst. Sci., 22, 5735-5739, https://doi.org/10.5194/hess-22-5735-2018, 2018.

Tauro, F., Selker, J., Van De Giesen, N., Abrate, T., Uijlenhoet, R., Porfiri, M., Manfreda, S., Caylor, K., Moramarco, T., Benveniste, J., Ciraolo, G., Estes, L., Domeneghetti, A., Perks, M. T., Corbari, C., Rabiei, E., Ravazzani, G., Bogena, H., Harfouche, A., Broccai, L., Maltese, A., Wickert, A., Tarpanelli, A., Good, S., Lopez Alcala, J. M., Petroselli, A., Cudennec, C., Blume, T., Hut, R., and Grimaldi, S.: Measurements and observations in the XXI century (MOXXI): innovation and multi-disciplinarity to sense the hydrological cycle, Hydrolog. Sci. J., 63, 169-196, https://doi.org/10.1080/02626667.2017.1420191, 2018.

UNESCO: Draft text of the UNESCO recommendation on Open Science, available at: https://en.unesco.org/ science-sustainable-future/open-science/recommendation, last access: 5 October 2021.

Young, G., Demuth, S., Mishra, A., and Cudennec, C.: Hydrological sciences and water security: An overview, Proc. IAHS, 366, 1-9, https://doi.org/10.5194/piahs-366-1-2015, 2015.

Yu, Z., Lu, C., Cai, J., Yu, D., Mahe, G., Mishra, A., Cudennec, C., Van Lanen, H. A. J., Orange, D., and Amani, A.: Preface: Hydrological processes and water security in a changing world, Proc. IAHS, 383, 3-4, https://doi.org/10.5194/piahs-383-3-2020, 2020. 